\title{
Proposta de método para estimação de tamanho de parcela para culturas agrícolas
}

\author{
Leandro Homrich Lorentz ${ }^{1}$, Ronaldo Erichsen ${ }^{2}$, Alessandro Dal'Col Lúcio ${ }^{3}$
}

\section{RESUMO}

Dentre as formas reconhecidas para redução do erro experimental, destaca-se o tamanho ótimo da parcela experimental para aplicação dos tratamentos, existindo vários métodos para sua estimação, baseados em diferentes princípios. O objetivo deste trabalho é propor um método alternativo para a estimação do tamanho ótimo de parcela, para culturas agrícolas, compará-lo com métodos clássicos para esse fim e identificar com quais parâmetros seus resultados estão correlacionados. Realizou-se a validação dos resultados, utilizando-se dados de quatro experimentos com plantas de pimentão, em que se avaliou a produção de frutos, acumulada nas várias colheitas, cultivado em estufa com cobertura plástica. Os resultados obtidos pelo método proposto, denominado Método da Máxima Distância, são coerentes, mas fracamente associados aos resultados observados pelos demais métodos clássicos. O tamanho ótimo da parcela, estimado pelo Método da Máxima Distância, é fortemente influenciado pelo índice de heterogeneidade da produção, que mede a correlação entre as produções de parcelas contíguas. Há, ainda, as características de ser de fácil aplicação, praticamente invariável à acumulação da produção em diversas colheitas, adequando-se para culturas com duas ou mais colheitas nas mesmas plantas, como em experimentos com culturas olerícolas ou forrageiras.

Palavras-chave: precisão experimental, planejamento de experimentos, índice de heterogeneidade, Capsicum annuum L., colheitas múltiplas.

\section{ABSTRACT}

\section{Proposal method for plot size estimation in crops}

Optimum plot size for the application of treatments is among the recognized methods to reduce the experimental error. There are several methods for plot estimation based on different principles. The objective of this paper was to propose an alternative method for the estimation of optimum plot size for crops, compare it with classical methods and to identify which parameters their results are correlated with. Fruit yield data from four experiments with sweet pepper cultivated in a greenhouse were used to validate the proposed method. It was evaluated the yield accumulated in several harvests. The results obtained by the proposed method, denominated Maximum Distance Method, are consistent, but weakly associated with the results obtained by other classical methods. The optimal plot size estimated by the Maximum Distance Method is strongly influenced by the heterogeneity index of production that measures the correlation between the productions in contiguous plots. It still has the characteristics of being easy to apply, remains practically unchanged in the cumulative productions, adapting to crops with two or more harvests in the same plants, as in vegetable crops or forage experiments.

Key words: experimental precision, planning of experiments, heterogeneity index, Capsicum annuиm L, multiple harvests.

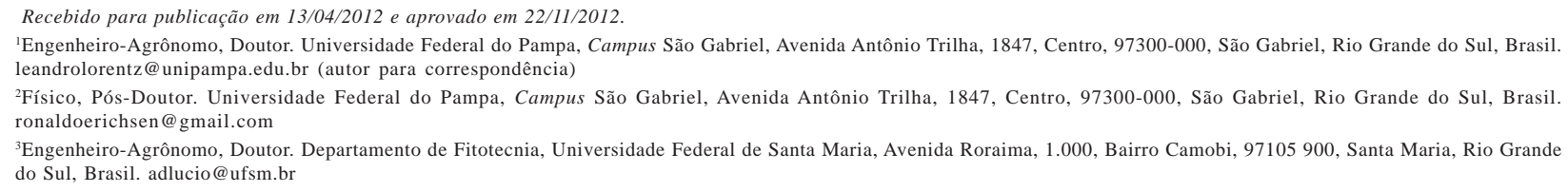




\section{INTRODUÇÃO}

O tamanho de parcela é um dos parâmetros experimentais reconhecidos pela literatura como forma de reduzir o erro experimental e está associado à escolha do delineamento adequado e ao uso de observações concomitantes (Steel et al., 1997). Sua determinação baseia-se na relação, notadamente inversa, entre o tamanho da parcela e a precisão do experimento. $\mathrm{O}$ aumento do tamanho da parcela, entretanto, não resulta em comportamento linear decrescente das medidas de variabilidade, como a variância ou o coeficiente de variação, mas, em função potencial negativa, pois ocorrem, inicialmente, melhorias relevantes na precisão com pequeno aumento do tamanho e, a partir de um determinado ponto $\left(\mathrm{X}_{0}\right)$, esse efeito se reduz gradativamente. Assim, o aumento do tamanho da parcela a partir desse ponto produz pouco incremento em precisão, como descrito em trabalho clássico de Smith (1938). A partir desse ponto, o aumento da precisão é obtido pelo uso de mais repetições.

Vários são os métodos que visam a estimar esse ponto e a indicá-lo como o tamanho ótimo da parcela $\left(\mathrm{X}_{0}\right)$. A literatura contempla diversos métodos para sua determinação, que se valem de vários princípios, como a identificação do ponto, de forma visual (Federer, 1955), por métodos algébricos (Lessman \& Atkins, 1963; Méier \& Lessman, 1971), com base em modelos de superfície de resposta (Barros \& Tavares, 1995), a partir de experimentos com aplicação de tratamentos (Rossetti, 2002; Alves \& Seraphin, 2004), por uso do coeficiente de autocorrelação parcial de primeira ordem (Paranaíba et al., 2009), ou, ainda, de regressão linear com resposta em plateau (Cargnelutti Filho et al, 2011).

Como já observado por Henriques Neto et al. (2004), Mello et al. (2004), Lopes et al. (2005), Donato et al. (2008) e Cargnelutti Filho et al. (2011), os diversos métodos de estimação do tamanho de parcela levam a diferentes resultados. Há diferenças mesmo quando os métodos baseiam-se em princípios semelhantes, como o da estimação do ponto de máxima curvatura da relação entre o coeficiente de variação ou variância, em função do tamanho de parcelas simuladas. Isso ocorre, principalmente, porque os métodos consideram de forma diferente as relações entre os parâmetros estimados para sua determinação.

Como alternativa aos métodos de estimação de tamanho de parcela, o objetivo deste trabalho é propor um método iterativo para estimativa do tamanho ótimo de parcela, compará-lo com resultados de métodos clássicos e identificar com quais parâmetros seus resultados são correlacionados.

\section{MATERIAL E MÉTODOS}

\section{Base teórica para o método}

O método proposto une os conceitos da Lei da Variância, proposta por Smith (1938), e o método da máxima curvatura, proposto por Federer (1955).

Para sua aplicação, é necessária a instalação e condução de um experimento em branco, com a cultura de interesse. Em seguida, subdivide-se a área experimental em porções menores, denominadas de unidades básicas, das quais são coletados os dados de forma independente, normalmente informações da produção, identificando a posição relativa (fila e parcela dentro da fila) da planta ou parcela na área experimental. Posteriormente à tomada dos dados, são simuladas parcelas de tamanhos e formas diferentes, por meio da soma da produção das parcelas contíguas. Federer (1955) orienta que, para cada tamanho simulado $(\mathrm{X})$, calcule-se o coeficiente de variação $\left(\mathrm{CV}_{\mathrm{x}}\right), \mathrm{o}$ qual deve ser colocado em um gráfico de dispersão em relação ao tamanho da respectiva parcela. Então, é traçada uma linha que represente a relação entre $\mathrm{X} \mathrm{e} \mathrm{CV}_{\mathrm{x}}$, para a qual espera-se que tenha a forma de um $\mathrm{J}$ invertido. Em seguida, por inspeção visual, identifica-se o ponto da máxima curvatura da relação, o qual indica o tamanho ótimo de parcela (Figura 1).

A Lei da Variância é baseada no pressuposto de que a heterogeneidade do solo pode ser medida por meio do desempenho das plantas, que tem por princípio a relação inversa entre uma medida de dispersão (como coeficiente de variação ou variância reduzida) dos valores obtidos nas parcelas de diferentes tamanhos e o respectivo tamanho de parcela. Essa relação node şer descrita por um modelo potencial negativo $V U_{x}=\frac{V_{1}}{X^{b}}$, em que $\mathrm{VU}_{\mathrm{x}}$ é a variância reduzida a uma unidade bâsica (planta), $\mathrm{V}_{1}$ é a estimativa da variância de uma unidade básica, X é o tamanho de parcela simulada e $b$ indica o índice de heterogeneidade do solo, que expressa a correlação entre as parcelas contíguas, variando entre zero e um (Smith, 1938).

O método propõe-se a encontrar o ponto da máxima distância entre a curva formada pelos valores estimados com base na relação de Smith (1938), cujo domínio compreende o intervalo entre o menor e o maior tamanho de parcela simulado, e uma reta gerada entre os pares ordenados de menor tamanho de parcela e respectivo $\mathrm{VU}_{\mathrm{x}} \mathrm{e}$ maior tamanho simulado e seu respectivo $\mathrm{VU}_{\mathrm{x}}$, conforme Figura 1, podendo $\mathrm{VU}_{\mathrm{x}}$ ser substituído por $\mathrm{CV}_{\mathrm{x}}$.

\section{Descrição do método da máxima distância (MMD)}

Para resolução desse problema, parte-se de geometria formada por uma curva, $\mathrm{y}_{\mathrm{C}}$, descrita por $y c=\frac{a}{X^{b}}$, e uma reta secante a esta curva, $y_{R}$, dada por $y_{R}=c x+d$, em que 
a e b podem ser os parâmetros $V_{1}$ e b (Smith, 1938), que originará o tamanho ótimo de parcela, pelo método denominado $\mathrm{MMDV}_{1}$ ou A e B (Lessman \& Atkins, 1963), resultando no tamanho ótimo pelo método MMDCV.

Procura-se o ponto da curva $\mathrm{y}_{\mathrm{C}}$ que esteja à maior distância da reta $y_{R}$, desde que o segmento de reta ao longo dessa distância seja perpendicular à reta $\mathrm{y}_{\mathrm{R}}$.

O método de solução aqui apresentado propõe expressar a reta perpendicular à reta $\mathrm{y}_{\mathrm{R}}$, como auxílio para encontrar o ponto procurado da curva $\mathrm{y}_{\mathrm{C}}$. Assim, chamarse-á $\mathrm{y}_{\mathrm{P}}$ esta reta perpendicular à reta $\mathrm{y}_{\mathrm{R}}$, expressa por $\mathrm{y}_{\mathrm{P}}=$ $\mathrm{ex}+\mathrm{f}$.

O coeficiente angular c e o coeficiente linear d, ambos da reta $y_{R}$, são fixos, e podem ser obtidos a partir dos dois pontos de $\mathrm{y}_{\mathrm{R}}$ comuns à curva $\mathrm{y}_{\mathrm{C}}$.

Sendo o ponto em comum entre $\mathrm{y}_{\mathrm{C}}$ e $\mathrm{y}_{\mathrm{R}}$, que está mais à esquerda, dado por $\left(\mathrm{x}_{\mathrm{CRi}}, \mathrm{y}_{\mathrm{CRi}}\right)$, e o ponto em comum mais à direita, dado por $\left(\mathrm{x}_{\mathrm{CRf}}, \mathrm{y}_{\mathrm{CRf}}\right)$, então c e d são expressos, respectivamente, por $c=\frac{y_{C R f}-y_{C R i}}{x_{C R f}-x_{C R i}} \mathrm{ed}=\mathrm{y}_{\mathrm{CRi}}-\mathrm{cx}_{\mathrm{Cri}}$, ou d $=\mathrm{y}_{\mathrm{CRf}}-\mathrm{cx}_{\mathrm{CRf}}$, sendo estas expressões para d obtidas, isolando-o na equação para $\mathrm{y}_{\mathrm{R}}$, tendo sido substituído, nesta, o ponto $\left(\mathrm{x}_{\mathrm{CRi}}, \mathrm{y}_{\mathrm{CRi}}\right)$, ou o ponto $\left(\mathrm{x}_{\mathrm{CRf}}, \mathrm{y}_{\mathrm{CRf}}\right)$.

$O$ coeficiente angular e da reta $\mathrm{y}_{\mathrm{P}}$ também é fixo e pode ser obtido, usando-se a condição de que as retas $y_{R}$ e $y_{P}$ sejam perpendiculares entre si. Desta maneira, $e=\frac{-1}{c}$.
A determinação do coeficiente linear $f$ da reta $y_{p}$ faz parte do método iterativo proposto como parte do objetivo deste trabalho. Este método tem as seguintes etapas:

1) Tomam-se determinados valores sobre o eixo dos $x$, situados entre os valores mencionados $\mathrm{x}_{\mathrm{CRi}}$ e $\mathrm{x}_{\mathrm{CRf}}$. Se esses valores sobre o eixo $\mathrm{x}$ forem igualmente espaçados, então este espaçamento é dado por $\Delta=\frac{x_{C R f}-x_{C R i}}{n}$, em que n é o número de intervalos em que será dividido o domínio da função (por exemplo, 10000 intervalos).

2) Para um valor determinado sobre o eixo $x$, p.e., $x_{j}$, calcula-se $\mathrm{y}_{\mathrm{C}}\left(\mathrm{x}_{\mathrm{j}}\right)$ e faz-se $\mathrm{y}_{\mathrm{C}}\left(\mathrm{x}_{\mathrm{j}}\right)=\mathrm{y}_{\mathrm{P}}\left(\mathrm{x}_{\mathrm{j}}\right)$, ou seja, a reta $\mathrm{y}_{\mathrm{P}}$, perpendicular à reta $\mathrm{y}_{\mathrm{R}}$, intercepta a curva $\mathrm{y}_{\mathrm{C}} \mathrm{em} \mathrm{x}_{\mathrm{j}}$. Os valores $\mathrm{y}_{\mathrm{C}}\left(\mathrm{x}_{\mathrm{j}}\right)$ e $\mathrm{y}_{\mathrm{P}}\left(\mathrm{x}_{\mathrm{j}}\right)$ doravante serão referidos como $\mathrm{y}_{\mathrm{Cj}}$ e $\mathrm{y}_{\mathrm{Pj}}$.

3) Obtém-se f, isolando-o na equação para $y_{P}$, tendo sido substituído nela o ponto $\left(\mathrm{x}_{\mathrm{j}}, \mathrm{y}_{\mathrm{Pj}}\right)$, de forma que $\mathrm{f}=\mathrm{y}_{\mathrm{Pj}}$ $-e_{x j}$, o que proporciona conhecer completamente a reta $y_{P}$.

4) Obtém-se o ponto em comum entre $y_{R}$ e $y_{P j}$, $\left(x_{R P j}\right.$, $\mathrm{y}_{\mathrm{RPj}}$ ), resolvendo-se o sistema $\left\{\begin{array}{l}y_{R P j}=c x_{R P_{j}}+d \\ y_{R P j}=e x_{R P_{j}}+f\end{array}\right.$, o qual tem como solução $x_{R P j}=\frac{t-d}{c-e}$.

5) Calcula-se a distância entre os pontos $\left(x_{\mathrm{Cj}}, \mathrm{y}_{\mathrm{Cj}}\right)$ e $\left(\mathrm{x}_{\mathrm{RPj}}, \mathrm{y}_{\mathrm{RPj}}\right)$, distância esta sobre a reta $\mathrm{y}_{\mathrm{Pj}}$, a qual é perpendicular a $\mathrm{y}_{\mathrm{R}}$, e é dada por $\mathrm{d}_{\mathrm{CR}}=\sqrt{\left(\mathrm{yc}_{\mathrm{j}}-\mathrm{y}_{\mathrm{RPj}}\right)^{2}+\left(\mathrm{xc}_{\mathrm{j}}-\mathrm{x}_{\mathrm{RPj}}\right)^{2}}$.

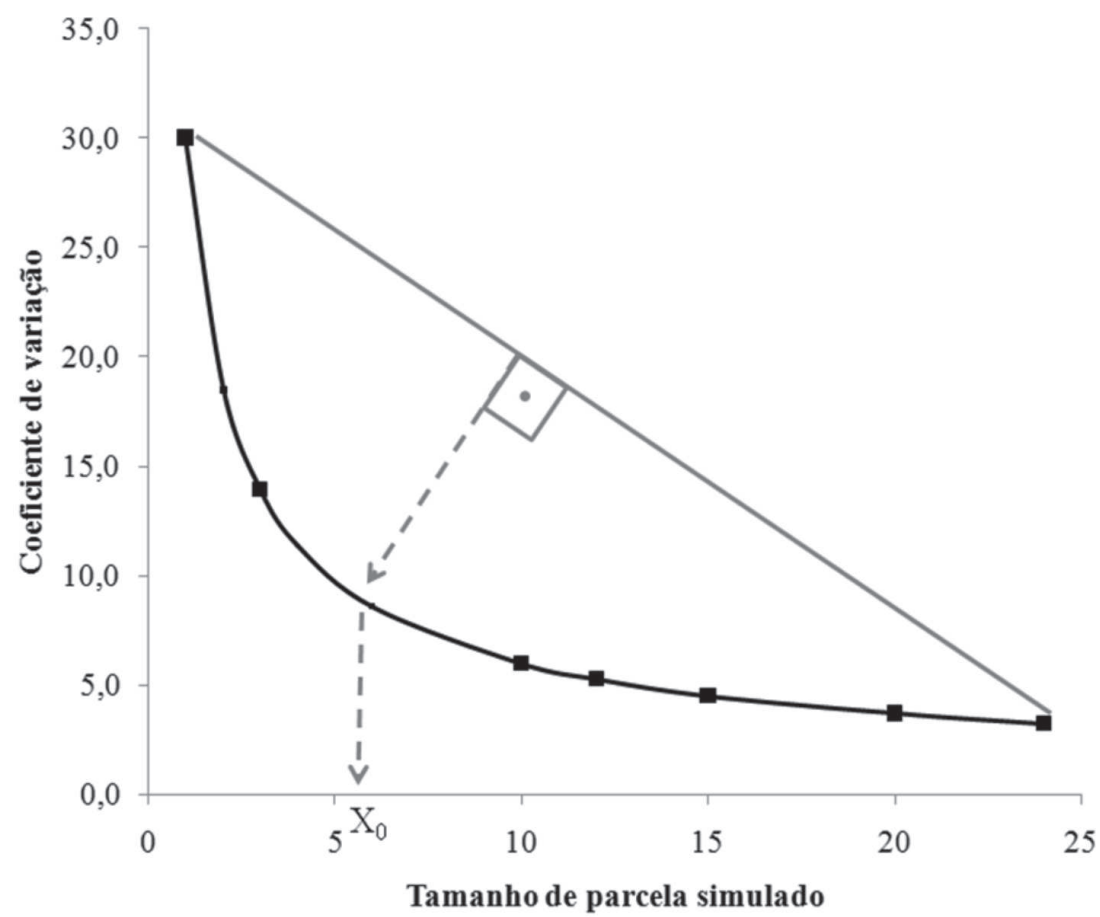

Figura 1. Representação esquemática do ponto a ser estimado como tamanho ótimo de parcela pelo Método da Máxima Distância. 
6) Repetindo-se as etapas de (2) a (5) para $x_{j}$ variando de $\mathrm{x}_{1}$ a $\mathrm{x}_{\mathrm{n}}$ (percorrendo todo o intervalo de interesse sobre o eixo $x$, de $x_{C i}$ a $x_{C f}$ ), armazenam-se, dos valores de $d_{C R}$ calculados, o maior deles $\left(\mathrm{d}_{\mathrm{CRmáx}}\right)$ e as correspondentes coordenadas do ponto sobre a curva $\mathrm{y}_{\mathrm{C}},\left(\mathrm{x}_{\mathrm{Cmáx}}, \mathrm{y}_{\mathrm{Cmáx}}\right)$. Esse valor máximo $\mathrm{d}_{\text {CRmáx }}$ é a distância máxima entre a curva dada por $\mathrm{y}_{\mathrm{C}}$ e a reta $\mathrm{y}_{\mathrm{R}}$, distância esta sobre um segmento de reta perpendicular a $\mathrm{y}_{\mathrm{R}}$.

A esta distância $\mathrm{d}_{\text {CRmáx }}$ corresponde um ponto ( $\mathrm{x}_{\text {Cmáx }}$, $\mathrm{y}_{\text {Cmáx }}$ ) sobre a curva $\mathrm{y}_{\mathrm{C}}$, o qual fica, assim, determinado, e é a solução do problema a que se propôs aqui resolver.

A programação para obtenção do tamanho ótimo de parcela pelos métodos propostos está disponibilizada na Tabela 4, utilizando-se o SAS Enterprises Guide 4.2. As informações que devem ser fornecidas para sua execução são: os pares $\mathrm{V}_{1}$ e b ou A e B e o intervalo do domínio da função, dado pelo menor tamanho simulado (p.e. $x c i=1) \mathrm{e}$ maior tamanho de parcela simulado (p.e. $x c f=24)$, e o número de espaçamentos para subdividir-se o domínio da função (p.e. imax = 10000). O resultado é o tamanho ótimo, pelos métodos $\mathrm{MMDV}_{1}$ ou MMDCV, apresentado diretamente no output do software.

\section{Validação com dados reais}

Para a validação dos resultados, utilizaram-se dados provenientes de quatro experimentos em branco, com a cultura do pimentão em cultivo protegido, implantados durante as estações de cultivo verão/outono de 2001 e 2002, nos quais foram realizadas cinco e quatro colheitas, respectivamente; e na estação inverno/primavera dos anos de 2001 e 2003, em que foram realizadas oito e quatro colheitas, respectivamente. Todos os experimentos foram conduzidos no Departamento de Fitotecnia da Universidade Federal de Santa Maria, em estufas de dimensões 24 x 10m, cobertas por filme de polietileno de baixa densidade, de 100 micras de espessura, situadas nas coordenadas 2943'23' S e $53^{\circ} 43^{\prime} 15^{\prime \prime} \mathrm{W}$ e altitude $95 \mathrm{~m}$. Mais detalhes sobre tratos culturais, datas de implantação, infraestrutura, etc., podem ser obtidos em Lorentz (2008).

A área experimental total era composta por dez linhas de cultivo, orientadas no sentido Norte-Sul, com 70 plantas cada. Subdividiu-se a área em duas partes (lados leste e oeste), pois, segundo Lúcio et al. (2003) e Mello et al. (2004), o interior da estufa plástica constitui-se de um ambiente heterogêneo e a subdivisão foi uma tentativa de reduzir a heterogeneidade, oriunda da incidência solar, nas laterais do cultivo protegido ao longo do dia. Então, desprezaram-se as linhas de borda e as cinco primeiras plantas de cada linha, totalizando área útil de quatro linhas, de 60 plantas por lado, das quais se pesou a fitomassa fresca acumulada nas colheitas dos frutos de pimentão de cada planta, identificada a sua posição den- tro da linha. As plantas com produção nula nas colheitas receberam valor zero.

A partir das produções individuais das plantas, foram simulados 17 tamanhos distintos de parcelas (em número de plantas, X) com diferentes larguras (L) e comprimentos (C), obtidos pela soma da produção das plantas adjacentes, com os seguintes tamanhos: $1 * 1$ ( $\mathrm{X}=1$ planta), $1 * 2$ ( $\mathrm{X}=2$ plantas), $1 * 3$ ( $\mathrm{X}=3$ plantas), $1 * 4(\mathrm{X}=4$ plantas $)$, $1 * 6(\mathrm{X}=6$ plantas $), 1 * 10(\mathrm{X}=10$ plantas $), 1 * 12(\mathrm{X}=12$ plantas), $1 * 15(\mathrm{X}=15$ plantas $), 1 * 20(\mathrm{X}=20$ plantas $), 2 * 2$ ( $\mathrm{X}=4$ plantas), $2 * 3$ ( $\mathrm{X}=6$ plantas), $2 * 4(\mathrm{X}=8$ plantas $)$, $2 * 6(X=12$ plantas $), 2 * 10(X=20$ plantas $), 2 * 12(X=24$ plantas $), 4 * 4$ ( $\mathrm{X}=16$ plantas $), 4 * 6(\mathrm{X}=24$ plantas $)$, em que $\mathrm{X}=\mathrm{L} * \mathrm{C}$.

Para cada tamanho de parcela simulado, calcularam-se a média $\left(\mathrm{M}_{\mathrm{x}}\right)$, a variância $\left(\mathrm{V}_{\mathrm{x}}\right)$ e o número de repetições $\left(r_{x}=240 / X\right)$. A partir desses dados, calcularam-se variância reduzida para uma unidade básica dada por $V U_{x}=\frac{V_{x}}{X^{2}}$, o coeficiente de variação $C V_{x}=100 \frac{\sqrt{V_{x}}}{M_{x}}$ e os graus de liberdade $\left(\mathrm{GL}_{\mathrm{x}}=\mathrm{r}_{\mathrm{x}}-1\right)$.

A seguir, estimou-se o índice de heterogeneidade da produção (b), por meio da equação $\mathrm{VU}_{\mathrm{x}}=\frac{\mathrm{V}_{1}}{\mathrm{X}^{\mathrm{b}}}$ (Smith, 1938), em que $V_{1}$ é a estimativa da variância entre as parcelas de uma unidade básica. Analogamente à equação acima, estimaram-se os coeficientes A e B da função $\mathrm{CV}_{\mathrm{x}}=\frac{\mathrm{A}}{\mathrm{X}^{\mathrm{B}}}$, proposta por (Lessman \& Atkins, 1963), em que A estima o coeficiente de variação entre as parcelas de uma unidade básica e $\mathrm{B}=0,5 \mathrm{~b}$, ambas logaritmizadas e ponderadas pelos graus de liberdade associados aos respectivos tamanhos de parcela (Federer, 1955; Steel et al., 1997).

Realizou-se a comparação das equações geradas dentro de cada colheita e experimento para a mesma origem $\left(\mathrm{V}_{1}\right.$ e A) e mesma inclinação (b e B) entre os lados da estufa plástica, pelo método de Snedecor (Schneider 2009).

Calculou-se o tamanho da parcela também pelo método da regressão quadrática, com resposta com plateau $(R Q P)$, dado por $Y_{i}=\left\{\begin{array}{ll}\beta_{0}+\beta_{1} X+\beta_{2} X^{2} & \text { se } X \leq X_{0} \\ \beta_{0}+\beta_{1} X_{0}+\beta_{2} X_{0}^{2} & \text { se } X>X_{0}\end{array}\right.$, em que $\beta_{0}, \beta_{1}, \beta_{2}$ são parâmetros da equação quadrática, $X_{0}$ será o tamanho ótimo de parcela, estimado por esse método, que satisfaz a condição $X_{0}=\frac{-\beta_{1}}{2 \beta_{2}}$ (Kaps e Lamberson, 2004), considerando-se o ponto de mínima da equação, e pelo método da máxima curvatura modificado (MCM), conforme a equação $X_{0}=\left[\frac{A^{2} B^{2}(2 B+1)}{B+2}\right]^{\frac{1}{2 B+2}}$, em que $X_{0}$ 
representa o tamanho ótimo de parcela, em número de plantas, estimado pelo método (Méier \& Lessman, 1971).

Procedeu-se à análise de correlação linear simples, com $5 \%$ de probabilidade de erro, entre a média e a variância e coeficiente de variação, os coeficientes $\mathrm{V}_{1}, \mathrm{~b}, \mathrm{~A}, \mathrm{~B}$ e as estimativas do tamanho ótimo pelos métodos MCM, RQP, $\mathrm{MMDV}_{1}$ e MMDCV.

Para o cálculo das variâncias dos diferentes tamanhos de parcela, utilizou-se programação no aplicativo MSExcel, e, para as demais determinações, o software SAS Enterprise Guide 4.2 (SAS, 2008).

\section{RESULTADOS E DISCUSSÃO}

Observa-se que uma estufa com cobertura plástica, com plantas cultivadas no solo, constitui-se um ambiente altamente heterogêneo, corroborando os resultados de Lúcio et al. (2003), podendo ser verificado pelos elevados coeficientes de variação, pois $50 \%$ dos $\mathrm{CV}_{\mathrm{x}}$, apresentamse entre 30,0 e $50,0 \%$ e $28,57 \%$ dos $\mathrm{CV}_{\mathrm{x}}$ superiores a $100 \%$, mas com certa tendência a diminuir com o acúmulo da produção das colheitas (Tabela 1).

Observa-se que os coeficientes de determinação, relativos ao ajustamento da equação de Smith (1938) e Lessman \& Atkins (1963) logaritmizados, foram elevados, na maioria dos casos superiores a $90 \%$, indicando que os dados comportam-se como o esperado, ajustando a relação potencial negativa. Assim, infere-se que os 17 tamanhos de parcela simulados foram eficientes em captarem a correlação real entre a produção das plantas nas colheitas acumuladas.

Observa-se que o índice de heterogeneidade do solo (b), que reflete a correlação entre as parcelas contíguas, mostrou-se elevado, sendo $50 \%$ dos valores encontrados entre 0,800 e 1,000 e $12 \%$ superiores à unidade, indicando as que produções das parcelas vizinhas não são correlacionadas. Ou seja, além de ser um ambiente heterogêneo, essa heterogeneidade está dispersa entre as plantas, aleatoriamente. Verificou-se, ainda, que só não se constatou paralelismo dos índices de heterogeneidade (b), entre os lados leste e oeste, na primeira colheita dos cultivos realizados no verão/outono de 2001 e 2002 e na estação inverno/primavera de 2003, casos, estes, em que as plantas contíguas apresentaram variações em intensidades diferentes, entre os lados da estufa, o que se torna não significativo, a partir do acúmulo de duas ou mais colheitas.

Por meio do método da Máxima Curvatura Modificado (Méier \& Lessman, 1971), o tamanho recomendado variou entre seis e 13 plantas por parcela, dependendo do número de colheitas realizadas e da estação de cultivo das plantas (Tabela 2), como, por exemplo, no caso do Experimento 1, no qual seriam necessárias parcelas de 13 plantas, caso se optasse por uma única colheita, e sete plantas, caso o interesse fosse acumular cinco colheitas. Considerando-se os resultados baseados nesse método, o tamanho ótimo de parcela tende a ser menor, à medida que a produção das plantas acumula-se nas sucessivas colheitas, acompanhando a tendência do coeficiente de variação.

O método da regressão quadrática com resposta com plateau (RQP) apresentou respostas coerentes, com medidas variando, na maioria das vezes, entre dez e 13 plantas por parcela (Tabela 2), sem, entretanto, resultados significativamente correlacionados com os dos demais métodos avaliados, contrariando Cargnelutti Filho et al. (2011), os quais encontraram medidas altamente associadas com as estimativas geradas pelo MCM.

Pelo Método da Máxima Distância proposto (tanto o $\mathrm{MMDV}_{1}$ como o MMDCV), o tamanho da parcela estimado foi de cinco ou seis plantas por parcela, indiferentemente quanto às produções acumuladas nas várias colheitas (Tabela 2). Essa característica torna o método adequado para determinação do tamanho de parcela, para culturas em que são realizadas diversas colheitas nas mesmas plantas, ou medidas repetidas na mesma parcela, como as olerícolas ou forrageiras, sendo necessária a tomada dos dados apenas em um único momento, para se obter esse parâmetro, pois é esperado o mesmo valor estimado para as demais colheitas.

A fim de verificar quais os parâmetros responsáveis pela determinação do tamanho da parcela, investigou-se a correlação entre os tamanhos estimados pelos diferentes métodos e os parâmetros usados para seu cálculo e medidas descritivas (Tabela 3), a partir da matriz de correlação entre os dados apresentados na Tabela 1.

A estimativa do tamanho ótimo, pelo método da Máxima Curvatura Modificado (MCM), mostrou-se fortemente associada à variabilidade da produção da cultura, refletida pela estimativa do coeficiente de variação (A), com correlação de 0,99 , corroborando a magnitude da associação encontrada por Storck et al. (2006a) e Oliveira et al. (2006), para as culturas do milho e batata, respectivamente. No entanto, os resultados encontrados contrariaram os relatos, dos mesmos autores, em relação ao índice de heterogeneidade da produção, pois a correlação encontrada por eles foi positiva e alta entre esses atributos e, neste caso, foi negativa e não significativa $\left(\mathrm{r}_{\mathrm{MCMxb}}=-0,284\right)$.

Já os métodos propostos, tiveram associação praticamente perfeita e negativa $(-0,999)$ com o índice de heterogeneidade da produção (b), que reflete a correlação da produção observada entre as parcelas contíguas, sendo esse o principal determinante do resultado observado. Esse parâmetro, na equação de Smith (1938), modela a curvatura do decréscimo da variável resposta $\left(\mathrm{VU}_{\mathrm{x}}\right.$ ou $\mathrm{CV}_{\mathrm{x}}$ ), com o aumento do tamanho da parcela simulada $(\mathrm{X})$. 
Os valores gerados pelo Método da Máxima distân-

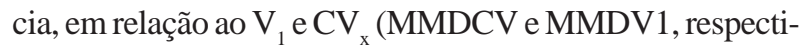
vamente), apresentaram correlação praticamente perfeita $(0,999)$ entre si, o que se justifica pelo fato de ambas basearem-se no mesmo princípio e por apresentarem alta correlação entre os índices b e B, com estimativas ligeiramente superiores, quando utilizados os parâmetros A e B.
O bom ajustamento das equações também é refletido, ao se relacionar o $\mathrm{CV}_{\mathrm{x}}$ observado e o $\mathrm{A}\left(\mathrm{CV}_{\mathrm{x}}\right.$ estimado pelas equações) e a Variância observada e a VU (Variância estimada pelas equações), que geraram correlação de 0,969 e 0,956 , respectivamente.

Frente aos resultados, podem-se observar algumas características do método proposto: a escolha pela esta-

Tabela 1. Coeficiente de Variação (CV\%), coeficientes $\mathrm{V}_{1}$ e b estimados dos modelos de Smith (1938) e A e B estimados pelo método de Lessman \& Atkins (1963), coeficiente de determinação ajustado $\left(\mathrm{r}^{2}\right)$ para produções de frutos de pimentão acumulados nas colheitas em ambos os lados da estufa

\begin{tabular}{|c|c|c|c|c|c|c|c|c|}
\hline Experimento & $\begin{array}{c}\text { Prod. } \\
\text { acumulada }\end{array}$ & Lado & CV\% & $\mathbf{V}_{1}$ & b & $\mathbf{A}$ & B & $\mathbf{r}^{2}$ \\
\hline 1 & $\mathrm{C} 1-\mathrm{C} 1$ & Leste & 93,4 & 0,026 & $0,872^{*}$ & 93,962 & $0,436^{*}$ & 0,97 \\
\hline 1 & $\mathrm{C} 1-\mathrm{C} 1$ & Oeste & 82,9 & 0,044 & 0,614 & 82,411 & 0,307 & 0,94 \\
\hline 1 & $\mathrm{C} 1-\mathrm{C} 2$ & Leste & 65,4 & 0,065 & $0,937^{\text {ns }}$ & 65,709 & 0,468 ns & 0,95 \\
\hline 1 & $\mathrm{C} 1-\mathrm{C} 2$ & Oeste & 45,3 & 0,068 & 1,057 & 46,255 & 0,528 & 0,97 \\
\hline 1 & $\mathrm{C} 1-\mathrm{C} 3$ & Leste & 46,0 & 0,093 & $0,988^{\text {ns }}$ & 46,007 & $0,494^{\mathrm{ns}}$ & 0,97 \\
\hline 1 & $\mathrm{C} 1-\mathrm{C} 3$ & Oeste & 38,0 & 0,089 & 1,044 & 38,917 & 0,522 & 0,98 \\
\hline 1 & $\mathrm{C} 1-\mathrm{C} 4$ & Leste & 44,1 & 0,122 & $0,932^{\mathrm{ns}}$ & 43,161 & $0,466^{\mathrm{ns}}$ & 0,96 \\
\hline 1 & $\mathrm{C} 1-\mathrm{C} 4$ & Oeste & 36,5 & 0,121 & 0,998 & 37,359 & 0,499 & 0,94 \\
\hline 1 & $\mathrm{C} 1-\mathrm{C} 5$ & Leste & 41,6 & 0,186 & $1,002^{\text {ns }}$ & 42,162 & $0,501^{\mathrm{ns}}$ & 0,95 \\
\hline 1 & $\mathrm{C} 1-\mathrm{C} 5$ & Oeste & 33,2 & 0,122 & 1,029 & 33,950 & 0,515 & 0,96 \\
\hline 2 & $\mathrm{C} 1-\mathrm{C} 1$ & Leste & 93,4 & 0,168 & $0,734^{\mathrm{ns}}$ & 68,581 & $0,367^{\mathrm{ns}}$ & 0,89 \\
\hline 2 & $\mathrm{C} 1-\mathrm{C} 1$ & Oeste & 82,9 & 0,085 & 0,752 & 101,387 & 0,376 & 0,93 \\
\hline 2 & $\mathrm{C} 1-\mathrm{C} 2$ & Leste & 51,9 & 0,188 & $0,894^{\text {ns }}$ & 51,615 & $0,447^{\mathrm{ns}}$ & 0,95 \\
\hline 2 & $\mathrm{C} 1-\mathrm{C} 2$ & Oeste & 62,0 & 0,197 & 1,013 & 63,276 & 0,507 & 0,97 \\
\hline 2 & $\mathrm{C} 1-\mathrm{C} 3$ & Leste & 45,1 & 0,266 & $0,923^{\text {ns }}$ & 45,007 & $0,462^{\mathrm{ns}}$ & 0,98 \\
\hline 2 & $\mathrm{C} 1-\mathrm{C} 3$ & Oeste & 44,3 & 0,257 & 0,969 & 44,194 & 0,485 & 0,99 \\
\hline 2 & $\mathrm{C} 1-\mathrm{C} 4$ & Leste & 43,3 & 0,313 & $0,931^{\mathrm{ns}}$ & 43,718 & $0,466^{\mathrm{ns}}$ & 0,97 \\
\hline 2 & $\mathrm{C} 1-\mathrm{C} 4$ & Oeste & 43,1 & 0,304 & 0,980 & 43,113 & 0,490 & 0,99 \\
\hline 3 & $\mathrm{C} 1-\mathrm{C} 1$ & Leste & 98,1 & 0,026 & $1,000^{*}$ & 100,076 & $0,500^{*}$ & 0,96 \\
\hline 3 & $\mathrm{C} 1-\mathrm{C} 1$ & Oeste & 80,9 & 0,042 & 0,531 & 80,651 & 0,265 & 0,95 \\
\hline 3 & $\mathrm{C} 1-\mathrm{C} 2$ & Leste & 109,3 & 0,017 & $0,663^{\text {ns }}$ & 104,361 & $0,331^{\mathrm{ns}}$ & 0,92 \\
\hline 3 & $\mathrm{C} 1-\mathrm{C} 2$ & Oeste & 105,6 & 0,017 & 0,714 & 105,057 & 0,357 & 0,93 \\
\hline 3 & $\mathrm{C} 1-\mathrm{C} 3$ & Leste & 81,5 & 0,027 & $0,679^{\text {ns }}$ & 78,378 & $0,339^{\mathrm{ns}}$ & 0,96 \\
\hline 3 & $\mathrm{C} 1-\mathrm{C} 3$ & Oeste & 80,9 & 0,026 & 0,768 & 79,553 & 0,384 & 0,93 \\
\hline 3 & $\mathrm{C} 1-\mathrm{C} 4$ & Leste & 54,6 & 0,041 & $0,892^{\text {ns }}$ & 52,944 & $0,446^{\mathrm{ns}}$ & 0,97 \\
\hline 3 & $\mathrm{C} 1-\mathrm{C} 4$ & Oeste & 66,1 & 0,052 & 0,740 & 66,896 & 0,370 & 0,84 \\
\hline 3 & $\mathrm{C} 1-\mathrm{C} 5$ & Leste & 51,5 & 0,049 & $0,842^{\text {ns }}$ & 49,932 & $0,4211^{\mathrm{ns}}$ & 0,96 \\
\hline 3 & $\mathrm{C} 1-\mathrm{C} 5$ & Oeste & 57,1 & 0,064 & 0,751 & 57,027 & 0,375 & 0,81 \\
\hline 3 & C1-C6 & Leste & 52,6 & 0,067 & $0,820^{\text {ns }}$ & 50,882 & $0,410^{\mathrm{ns}}$ & 0,95 \\
\hline 3 & C1-C6 & Oeste & 46,5 & 0,074 & 0,878 & 46,933 & 0,439 & 0,90 \\
\hline 3 & $\mathrm{C} 1-\mathrm{C} 7$ & Leste & 49,3 & 0,076 & $0,902^{\mathrm{ns}}$ & 48,081 & $0,451^{\mathrm{ns}}$ & 0,97 \\
\hline 3 & $\mathrm{C} 1-\mathrm{C} 7$ & Oeste & 45,6 & 0,081 & 0,830 & 46,027 & 0,415 & 0,93 \\
\hline 3 & $\mathrm{C} 1-\mathrm{C} 8$ & Leste & 47,3 & 0,093 & $0,988^{\text {ns }}$ & 45,632 & $0,494^{\mathrm{ns}}$ & 0,97 \\
\hline 3 & $\mathrm{C} 1-\mathrm{C} 8$ & Oeste & 43,1 & 0,094 & 0,920 & 43,283 & 0,460 & 0,92 \\
\hline 4 & $\mathrm{C} 1-\mathrm{C} 1$ & Leste & 93,4 & 0,075 & $0,818^{*}$ & 107,334 & $0,409^{*}$ & 0,90 \\
\hline 4 & $\mathrm{C} 1-\mathrm{C} 1$ & Oeste & 82,9 & 0,054 & 0,612 & 82,362 & 0,306 & 0,98 \\
\hline 4 & $\mathrm{C} 1-\mathrm{C} 2$ & Leste & 47,2 & 0,131 & $0,834^{*}$ & 48,386 & $0,417^{*}$ & 0,97 \\
\hline 4 & $\mathrm{C} 1-\mathrm{C} 2$ & Oeste & 52,8 & 0,126 & 0,685 & 52,506 & 0,343 & 0,96 \\
\hline 4 & $\mathrm{C} 1-\mathrm{C} 3$ & Leste & 39,2 & 0,192 & $0,691^{\text {ns }}$ & 39,852 & 0,346 & 0,97 \\
\hline 4 & $\mathrm{C} 1-\mathrm{C} 3$ & Oeste & 41,1 & 0,189 & 0,656 & 40,521 & 0,328 & 0,98 \\
\hline 4 & $\mathrm{C} 1-\mathrm{C} 4$ & Leste & 38,1 & 0,200 & $0,672^{\mathrm{ns}}$ & 38,578 & 0,336 & 0,97 \\
\hline 4 & $\mathrm{C} 1-\mathrm{C} 4$ & Oeste & 39,7 & 0,205 & 0,651 & 39,059 & 0,326 & 0,98 \\
\hline
\end{tabular}

${ }^{n s}$ Aceita-se a hipótese de paralelismo entre ambos os lados, com 5\% de probabilidade de erro. "Retas não paralelas entre ambos os lados. 
Tabela 2. Tamanho ótimo de parcela estimado pelos métodos da Máxima Curvatura Modificado (MCM), método da regressão quadrática com resposta com plateau (RQP) e métodos da máxima distância, utilizando os coeficientes $\mathrm{V}_{1}$ e b $\left(\mathrm{MMDV}_{1}\right)$ ou $\mathrm{A}$ e $\mathrm{B}$ (MMDCV)

\begin{tabular}{|c|c|c|c|c|c|c|c|c|c|}
\hline Exper & Método & C1C1 & $\mathrm{C} 1 \mathrm{C} 2$ & C1C3 & $\mathrm{C1C4}$ & C1C5 & C1C6 & C1C7 & C1C8 \\
\hline \multicolumn{10}{|c|}{ LADO LESTE } \\
\hline 1 & MCM & 12 & 9 & 7 & 7 & 7 & - & - & - \\
\hline 1 & MMDCV & 6 & 6 & 6 & 6 & 6 & - & - & - \\
\hline 1 & MMDV1 & 5 & 5 & 5 & 5 & 5 & - & - & - \\
\hline 1 & RQP & 9 & 10 & 9 & 8 & 9 & - & - & - \\
\hline 2 & MCM & 9 & 8 & 7 & 7 & - & - & - & - \\
\hline 2 & MMDCV & 6 & 6 & 6 & 6 & - & - & - & - \\
\hline 2 & MMDV1 & 5 & 5 & 5 & 5 & - & - & - & - \\
\hline 2 & RQP & 9 & 10 & 10 & 10 & - & - & - & - \\
\hline 3 & MCM & 13 & 13 & 10 & 8 & 8 & 8 & 8 & 7 \\
\hline 3 & MMDCV & 6 & 6 & 6 & 6 & 6 & 6 & 6 & 6 \\
\hline 3 & MMDV1 & 5 & 6 & 6 & 5 & 5 & 5 & 5 & 5 \\
\hline 3 & RQP & 11 & 7 & 8 & 8 & 9 & 8 & 9 & 8 \\
\hline 4 & MCM & 13 & 8 & 6 & 6 & - & - & - & - \\
\hline 4 & MMDCV & 6 & 6 & 6 & 6 & - & - & - & - \\
\hline 4 & MMDV1 & 5 & 5 & 6 & 6 & - & - & - & - \\
\hline 4 & RQP & 10 & 12 & 13 & 12 & - & - & - & - \\
\hline \multicolumn{10}{|c|}{ LADO OESTE } \\
\hline 1 & MCM & 10 & 8 & 7 & 7 & 6 & - & - & - \\
\hline 1 & MMDCV & 6 & 6 & 6 & 6 & 6 & - & - & - \\
\hline 1 & MMDV1 & 6 & 5 & 5 & 5 & 5 & - & - & - \\
\hline 1 & Plateau & 9 & 10 & 10 & 10 & 9 & - & - & - \\
\hline 2 & MCM & 13 & 9 & 7 & 7 & - & - & - & - \\
\hline 2 & MMDCV & 6 & 6 & 6 & 6 & - & - & - & - \\
\hline 2 & MMDV1 & 5 & 5 & 5 & 5 & - & - & - & - \\
\hline 2 & Plateau & 9 & 9 & 10 & 10 & - & - & - & - \\
\hline 3 & MCM & 10 & 13 & 11 & 9 & 8 & 7 & 7 & 7 \\
\hline 3 & MMDCV & 7 & 6 & 6 & 6 & 6 & 6 & 6 & 6 \\
\hline 3 & MMDV1 & 6 & 5 & 5 & 5 & 5 & 5 & 5 & 5 \\
\hline 3 & Plateau & 10 & 10 & 9 & 10 & 9 & 9 & 10 & 9 \\
\hline 4 & MCM & 10 & 8 & 6 & 6 & - & - & - & - \\
\hline 4 & MMDCV & 6 & 6 & 6 & 6 & - & - & - & - \\
\hline 4 & MMDV1 & 6 & 6 & 6 & 6 & - & - & - & - \\
\hline 4 & Plateau & 9 & 10 & 11 & 10 & - & - & - & - \\
\hline
\end{tabular}

Tabela 3. Correlação entre as estatísticas média, variância e coeficiente de variação $\left(M_{x}, V_{x}\right.$ e $C V_{x}$, respectivamente), coeficientes $V_{1}$ e b Smith (1938) e A e B Lessman \& Atkins (1963) e o tamanho ótimo de parcela estimado pelos métodos da Máxima Curvatura Modificado (MCM), método da regressão quadrática com resposta com plateau (RQP) e métodos da máxima distância, utilizando $\mathrm{V}_{1}$ e b $\left(M_{1}\right)$ e A e B (MMDCV)

\begin{tabular}{|c|c|c|c|c|c|c|c|c|c|c|}
\hline & $\mathbf{V}_{\mathrm{x}}{ }^{1}$ & $\mathrm{CV}_{\mathrm{x}}$ & $V_{1}$ & b & $\mathbf{A}$ & B & МCM & MMDV $_{1}$ & MMDCV & RQP \\
\hline$M_{x}$ & $0,928^{*}$ & $-0,849^{*}$ & $0,867^{*}$ & $0,337^{*}$ & $-0,823^{*}$ & $0,339^{*}$ & $-0,828^{*}$ & $-0,334^{*}$ & $-0,337^{*}$ & $0,441^{*}$ \\
\hline$V_{x}$ & & $-0,653^{*}$ & $0,956^{*}$ & $0,263^{\text {ns }}$ & $-0,631^{*}$ & $0,265^{\mathrm{ns}}$ & $-0,633^{*}$ & $-0,262^{\mathrm{ns}}$ & $-0,265^{\text {ns }}$ & $0,374^{*}$ \\
\hline $\mathrm{CV}_{\mathrm{x}}$ & & & $-0,564^{*}$ & $-0,436^{*}$ & $0,969^{*}$ & $-0,437^{*}$ & $0,956^{*}$ & $0,434^{*}$ & $0,437^{*}$ & $-0,298^{\mathrm{n}}$ \\
\hline $\mathrm{V}_{1}$ & & & & $0,234^{\text {ns }}$ & $-0,571^{*}$ & $0,237^{\mathrm{ns}}$ & $-0,570^{*}$ & $-0,235^{\mathrm{ns}}$ & $-0,236^{\mathrm{ns}}$ & $0,394^{*}$ \\
\hline $\mathrm{b}$ & & & & & $-0,401^{*}$ & $1,000^{*}$ & $-0,284^{*}$ & $-0,999^{*}$ & $-0,999^{*}$ & $-0,079^{\mathrm{n}}$ \\
\hline A & & & & & & $-0,402^{*}$ & $0,990^{*}$ & $0,399^{*}$ & $0,401^{*}$ & $-0,254^{\mathrm{n}}$ \\
\hline B & & & & & & & $-0,285^{\mathrm{ns}}$ & $-0,999^{*}$ & $-0,999^{*}$ & $-0,078^{\mathrm{n}}$ \\
\hline $\mathrm{MCM}$ & & & & & & & & $0,280^{\mathrm{ns}}$ & $0,284^{\mathrm{ns}}$ & $-0,300^{\mathrm{n}}$ \\
\hline $\mathrm{MMDV}_{1}$ & & & & & & & & & $0,999^{*}$ & $0,083^{\text {ns }}$ \\
\hline MMDCV & & & & & & & & & & $0,079^{\text {ns }}$ \\
\hline
\end{tabular}

${ }^{\text {ns }}$ Coeficiente de correlação de Pearson não significativo a 5\% de probabilidade de erro; ${ }^{*}$ Correlação significativa a 5\%.

Rev. Ceres, Viçosa, v. 59, n.6, p. 772-780, nov/dez, 2012 
Tabela 4. Rotina de programação para SAS Enterprises Guide 4.2.

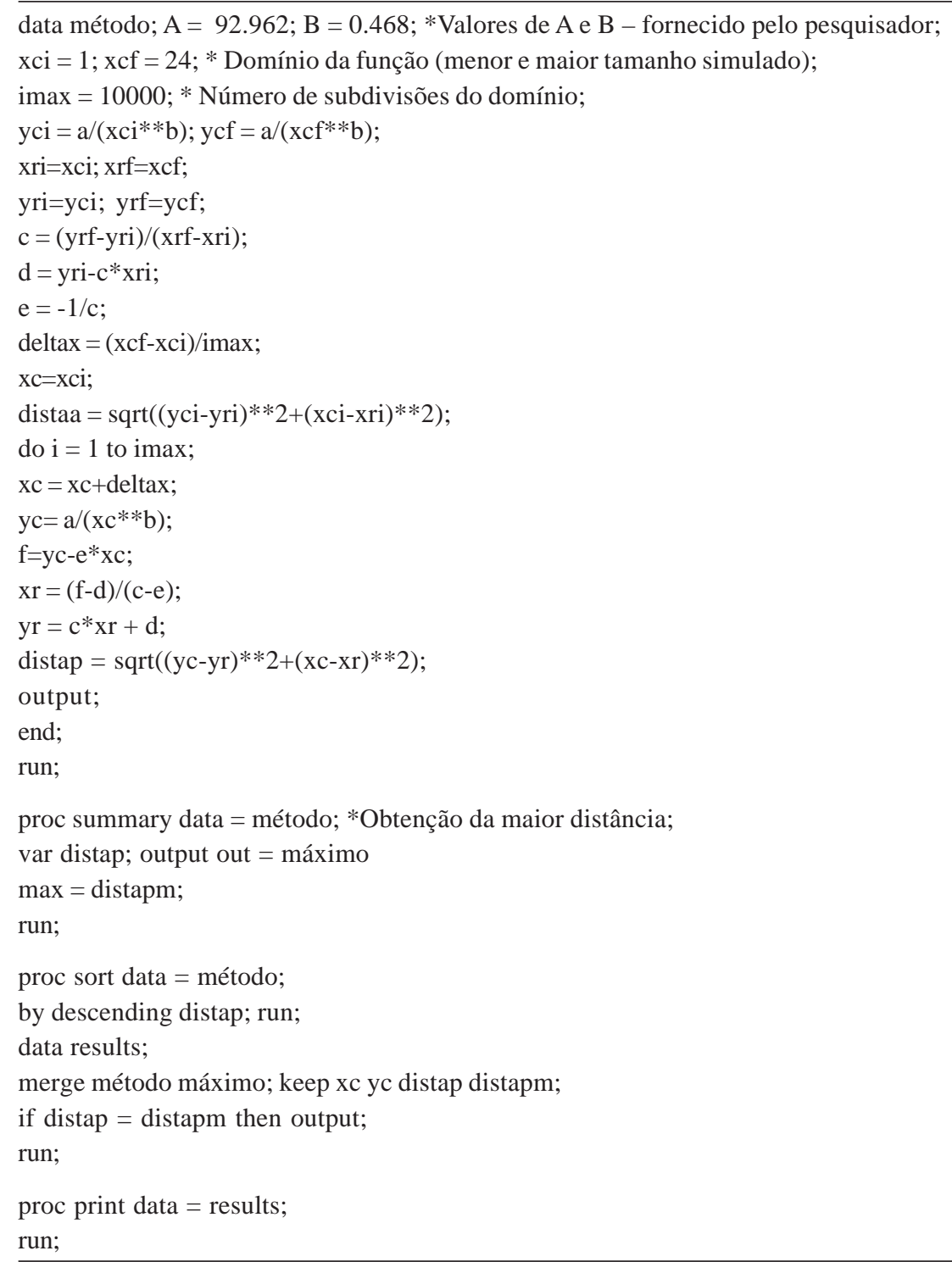

tística de dispersão $\mathrm{VU}_{\mathrm{x}}$ ou $\mathrm{CV}_{\mathrm{x}}$ influenciou a estimativa do tamanho ótimo da parcela, sendo preferido o uso do $\mathrm{CV}_{\mathrm{x}}$, associado aos parâmetros A e B para sua determinação, pois gerou-se resultado ligeiramente maior; apresentou-se estimativa estável de $\mathrm{X}_{0}$, mesmo nos casos em que se realizava o acúmulo da produção nas colheitas ou em condução de experimentos em épocas diferentes; mesmo que a cultura avaliada precise de várias colheitas para se obter a produção final, como as olerícolas, é necessário apenas uma colheita para determinação do tamanho ótimo, pois o resultado é estável, mesmo para produção acumulada em diversas colheitas.

\section{CONCLUSÕES}

O método apresenta resultados coerentes em relação aos demais métodos de estimativa de tamanho ótimo de parcela.
Os resultados do método são determinados, principalmente, pela magnitude do índice de heterogeneidade da produção.

\section{REFERÊNCIAS}

Alves SMF \& Seraphin JC (2004) Coeficiente de heterogeneidade do solo e tamanho de parcela. Pesquisa Agropecuária Brasileira, 39:105-111.

Barros I \& Tavares M (1995) Estimativa do tamanho ótimo de parcelas experimentais através de cálculos algébricos. Bragantia, 54:209-215.

Cargnelutti Filho A, Burin MTC, Casarotto G \& Lúcio AD (2011) Métodos de estimativa do tamanho ótimo de parcelas experimentais de híbridos de milho simples, triplo e duplo. Ciência Rural, 41:1509-1516.

Donato SLR, Siqueira DL, Silva SO, Cecon PR, Silva JA \& Salomão LCC (2008) Estimativas de tamanho de parcelas para avaliação de descritores fenotípicos em bananeira. Pesquisa Agropecuária Brasileira, 43:957-969. 
Federer WT (1955) Experimental design: theory and application. $3^{a}$ ed. Nova York, Oxford \& IBH Publishing. 593p.

Henriques Neto D, Sediyama T, Souza MA, Cecon PR, Yamanaka CH, Sediyama MAN \& Viana AE (2004) Tamanho de parcelas em experimentos com trigo irrigado sob plantio direto e convencional. Pesquisa Agropecuária Brasileira, 39:517-524.

Kaps M \& Lamberson WR (2004) Bioestatistics for animal science. Wallingford, CABI-PUBLISHING. 445p.

Lessman KJ \& Atkins RE (1963) Comparisons of planning arrangements and estimates of optimum hill plot for grain sorghum yield tests. Crop Science, 3:477-481.

Lopes SJ, Storck L, Lúcio AD, Lorentz LH, Lovato C \& Dias VO (2005) Tamanho de parcela para produtividade de grãos de sorgo granífero em diferentes densidades de plantas. Pesquisa Agropecuária Brasileira, 40:525-530.

Lorentz LH (2008) Relação entre estatísticas do planejamento experimental e tamanho de parcela para pimentão em estufa plástica. Tese de Doutorado. Universidade Federal de Santa Maria, Santa Maria. 72p.

Lúcio AD, Souza MF, Heldwein AB, Lieberknecht D, Carpes RH \& Carvalho MP (2003) Tamanho da amostra e método de amostragem para avaliação de características do pimentão em estufa plástica. Horticultura Brasileira 21:181-185.

Méier VD \& Lessman KJ (1971) Estimation of optimum field plot shape and size for testing yield in Crambe abssinica. Crop Science, 11:648-650.
Mello RM, Lúcio AD, Storck L, Lorentz LH, Carpes RH \& Boligon AA (2004) Size and form of plots for the culture of the italian pumpkin in plastic greenhouse. Scientia Agricola, 61:457-461.

Oliveira SJR, Storck L, Lúcio AD, Lopes SJ \& Martini LFD (2006) Índice de heterogeneidade, coeficiente de variação e tamanho ótimo de parcela em batata. Ciência Rural, 36:1710-1716.

Paranaíba PF, Ferreira DF \& Moraes AR (2009) Tamanho ótimo de parcelas experimentais: proposição de métodos de estimação. Revista Brasileira de Biometria, 27:255-268.

Rossetti AG (2002) Influência da área da parcela e do número de repetições na precisão de experimentos com arbóreas. Pesquisa Agropecuária Brasileira, 37:433-438.

SAS Institute Inc. (2008) Enterprises Guide. Version 4.2. Cary, Statistical Analysis System Institute.

Schneider PR (2009) Análise de regressão aplicada à engenharia florestal. Santa Maria, UFSM. 236p.

Smith HF (1938) An empirical law describing heterogeneity in yields of agricultural crops. Journal Agricultural Science, 28:01-23.

Steel RGD, Torrie JH \& Dickey DA (1997) Principles and procedures of statistics: a biometrical approach. New York, McGraw-Hill. 663p.

Storck L, Bisognin DA \& Oliveira SJE (2006) Dimensões dos ensaios e estimativas do tamanho ótimo de parcela em batata. Pesquisa Agropecuária Brasileira, 41:903-909. 